Proceedings of the 1998 IEEE

International Conference on Robotics \& Automation

Leuven, Belgium • May 1998

\title{
Landmark Perception Planning for Mobile Robot Localization
}

\author{
J. M. Armingol, L. Moreno, A. de la Escalera and M. A. Salichs \\ Universidad Carlos III de Madrid, Division of Systems Engineering and Automation. \\ C/ Butarque 15, 28911 Leganés (Madrid) SPAIN
}

\begin{abstract}
This paper presents a fuzzy perception planner that takes into account the time cost, the suitability of every landmark detection and the different uncertainties the robot encounters along its path for mobile robot localization. The sensor used is a camera with a motorized zoom on a pan \& tilt platform and the artificial landmarks are circles detected through normalized grayscale correlation. An Extended Kalman Filter is used to correct the position and orientation of the vehicle. The resulting self-localization module has been integrated successfully in a more complicated novigation system.
\end{abstract}

\section{Introduction}

A prerequisite for geometric navigation of a mobile robot is a position-finding method. A rough estimation of the robot location (position and orientation) is possible with odometry data. To reduce the uncertainty and error of the estimated location, relocalization techniques have been developed. Two approaches are used: the matching between the sensorial information and a map of the environment, or the detection of natural or artificial landmarks. In [3], the robot position is estimated by matching sensed features against a geometrical model of the environment using stereoscopy vision. In [6], a combination of a laser diode and a CCD camera is used; the sensorial information is modeled as straight lines that will be matched with a prior map of the environment. The second approach is the one used in this paper. Two independent problems have to be addressed: the shape, color and others landmark features in order to detect them and, secondly, how to select the best landmark to optimized the relocalization process. Several landmarks can be found in recent literature: Ricotti and Liotta [14] use a guide mark that consists of two elements: two circles and identification codes. They are designing a landmark that can be easily characterized by geometric features invariant in respect of scale factors and perspective views.
D'Orazio et al [5] use a landmark that consists of panels on which two groups of four LEDs were fixed. The camera can see the confidence points at any moment and they can be located easily in the image plane because of their brightness and arrangement.

Perception planning is a less studied field. It is necessary because the resultant location error depends on the amount and quality of the incoming sensor data. The robot has to drive in such a way that spatial features and artificial landmarks can be recognized optimally by its sensors. If the sensors loose contact for a long time, location uncertainty would grow and the robot will ultimately become lost. In order to keep stable the process, a planner must actively supervise the data acquisition and landmark extraction for the localization process. Perception planning is in charge of landmark selection during robot localization process, in order to get always a suitable landmark in a way that the robot has not to be stopped, or if it stops, for a time interval as short as possible, according to uncertainty criteria used in the position by system supervisor.

A first approach to this problem could be to consider a priori the mark selection [4], so that it has been carried out previous study of visibility intervals for any of the marks, which number is high enough to each path can be fully covered by the interval corresponding the whole marks. Once carried out the study, it must be selected the set of marks that robot will have to sense and follow along the path, so that sensory cost was minimum. This process can be carried out through uniform cost algorithms (all landmarks have equal cost of detection and tracking) or balanced cost. A landmark-based navigation system is described in [10], assuming landmark regions scattered across the workspace. In order to keep the location uncertainty low, a planner computes a guaranteed path from a start to a goal position via the known landmark regions. In [11], it is proposed a planning that assumes the existence of areas called islands of perfection where position sensing and motion control are accurate. 


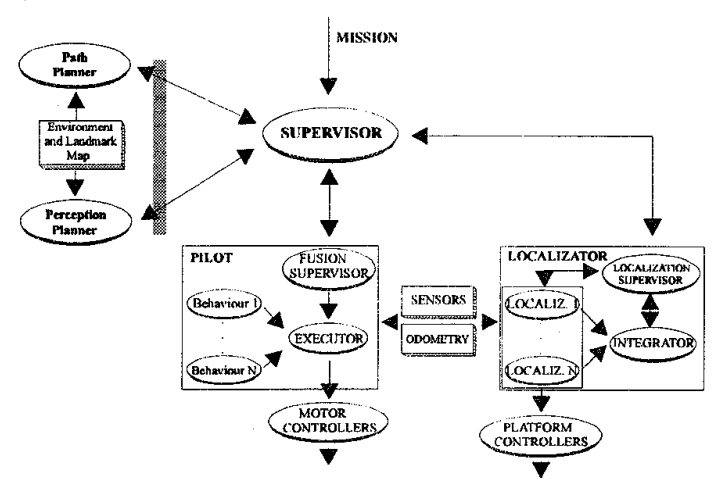

Figure 1. Mobile robot architecture.

Another possibility would be to make the path planning action conditional according to suitable mark selection to the localization process [1], it would be necessary to include a sequence of maneuvers assigned to make easy the localization process.

\section{Relocalization Planning}

In this paper, we propose a relocalization planner whose impact over the rest of control architecture elements were the less possible. The planner produces a preselection of landmarks on each area where the robot will go through. The architecture of the system (Fig. 1) uses a hybrid architecture (AFREB) approach with a wide range of reactive control methodologies [15][13]. AFREB architecture consists of the following modules: fusion supervisor, behaviour primitives, and executor.

The relocalization planner can be considered as an independent module that is run in parallel with the others. Their performance is based on the study of fixed control positions belonging to the path, and the combination of a preliminary landmark selection to carry out the localization in motion. In addition, the localization can also be performed with an optimum landmark selection with the vehicle stopped.

The selection function used is associated to the

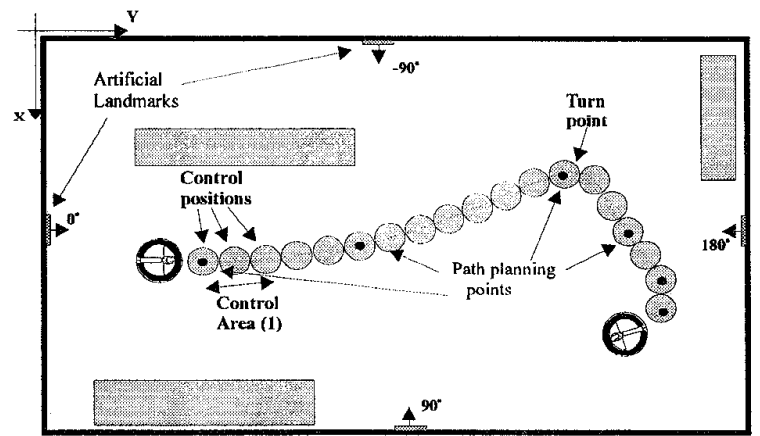

Figure 2. Modelling of a possible path. analysis of two cost functions:

- Deceleration cost of robot motion. The landmarks selection must be carried out in a way that every moment has a suitable mark in order to avoid the robot stop during localization process or during the shorter time interval.

- Mark acquisition cost. Associated to the movements involved in the image acquisition (a pan-tilt platform and a motorized zoom lens).

\subsection{Path Modelling}

If we consider that landmarks preselection is performed off line, it will be necessary to know which are the aim points produced by path planning, according to the mission proposed by the user. The proposed planning module interpolates intermediate successions of new points, that guarantee control positions separated by a maximum programmable length. Experimentally, it has been observed that the points with sudden leaps of vehicle orientation, the overall movement conjunction leads to increase of acquisition cost. These are labelled as turn points, not considering as control position and therefore excluded from the following analysis.

In the Fig. 2, the modelling of a possible path is shown. As a result of this modelling process, a set of control positions is generated, where it would be necessary to study the visibility conditions of the landmarks. The control positions are defined as circles of $0.5 \mathrm{~m}$ radius, placed on the path each $1 \mathrm{~m}$. At the same time, these control positions are grouped in areas of uniform distance to the landmarks, contained in an approximate length of $3 \mathrm{~m}$ (control area). The optical placement of zoom is the slower activity in relocalization process time, in this way, it is expected to set uniform focal distance areas that group different landmarks, to decrease the acquisition cost.

\subsection{Visual Suitability of Landmarks}

The proposed selection method has been developed using fuzzy techniques. In order to evaluate the visual suitability of each landmark, at each control position, a scheme of fuzzy supervisor is used where the inputs are the estimated distance to the landmark $\left(\mathrm{d}_{\mathrm{i}}\right)$ and the vision angle $\left(\delta_{i}\right)$. The output will be a suitability value $\left(I_{i}\right)$ corresponding to the mark observation from a control position (Fig. 3). Landmarks with a low suitability value are rejected.

\subsection{Preselecting Landmarks}

The perception planner selects the best landmarks, at each control area, according to the following criteria: 


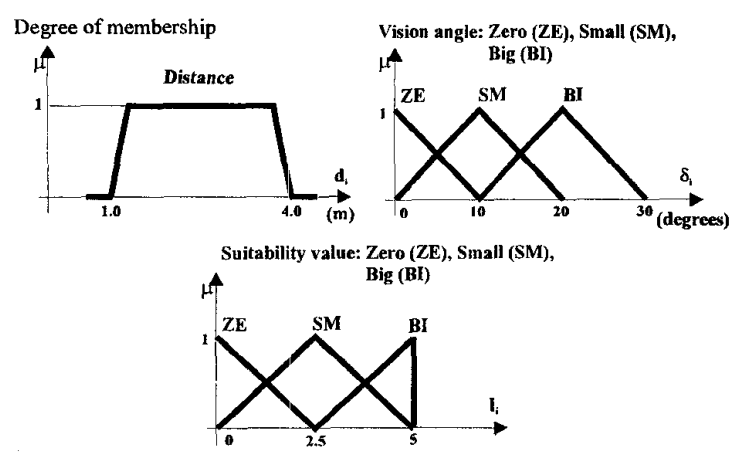

Figure 3. Selecting landmarks for each control position.

- Visual suitability

- They maintain their use in the whole control area, and can reduce the uncertainty at different axes.

- They are within the margins of a predetermined distance which guarantees the landmark visibility without optic movement.

The process performed on the visible landmarks, applying the previous criteria has the following steps:

- Landmarks are grouped with a fuzzy system, according to distance between the video camera and the landmark. A different setting of the zoom corresponds to each group.

- Landmarks with the highest geometric suitability to reduce uncertainty in $x$ and $y$ axes are determined.

\section{Plan Execution}

The relocalization plan can be executed in two different ways according to the uncertainty increase of robot position. Experimentally, two limits have been stablished in the position uncertainty. The robot intends to perform the relocalization process in motion, according to the landmarks selected in each control area, when the first limit has been reached. If the system were not able to identify the landmarks, the uncertainty of the position would increase and reach the second limit. In this case, the vehicle would be forced to stop, to allow an easier

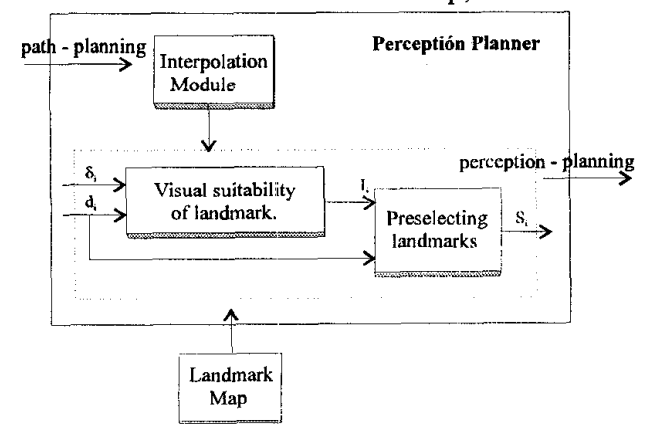

Figure 4. Perception planner scheme. acquisition and identification of the landmark. When the uncertainty decreases, the vehicle would restart again, beginning again the process in a cyclic way.

\subsection{Landmark Selection}

The acquisition process of the image is subject to a motion estimate of robot in the following cycles, as soon as the optic was adjusted to the selected value in the actual control area. In order to carry out the motion estimate the system have two mainly functions:

- relate the mobile robot position and orientation (odometry information),

- relate the mobile robot internal state (linear and rotational speed).

The time interval used is variable, depending on the time necessary for the move of the pan-tilt platform.

The landmark selection process is related to the actual value of uncertainty in the vehicle position. In order to perform this selection, a fuzzy supervisor has been developed according to Fig. 5. The input of this fuzzy module are the landmark orientation in terms of reference system $\left(\theta_{i}\right)$ and the localization uncertainty of both axes (P). As an output the supervisor gives the selected landmarks suitability for the given control area $\left(\mathrm{I}_{i}\right)$. When relocalization is performed with the robot stopped, the algorithm is different. The best landmark is selected according to the following criteria:

- it should allow a better adjustment of the motorized zoom lens, in order to guarantee the landmark detection.

- if the uncertainty of the vehicle position is very high, it is necessary to carry out the relocalization process according to the landmark belonging to both axes.

\section{Landmark Detection}

The localization system is based on the detection of artificial landmarks (solid circles place on a wall). The image of the landmark provides information regarding the robot's position and heading direction. The advantage of

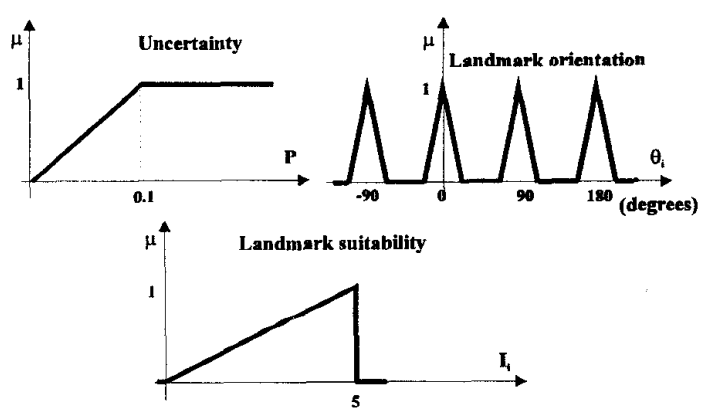

Figure 5. Landmark selection process. 
using artificial landmarks is that they can be designed in such a way that they will be easily recognized in the environment. In [8], they use a solid circle in black with two interior white dots to obtain the position of the mobile robot. A similar landmark is used in [7], two concentric circles that produce in the sensor image an elliptical edge.

Normalized grayscale correlation is a widely used method in industry for pattern matching applications. The correlation operation can be seen as a form of convolution where the pattern matching model $M(x, y)$ is analogous to the convolution kernel:

$$
\begin{aligned}
r(x, y)=I(x, y) & \circ M(x, y)= \\
& \sum_{i=0}^{i=N} \sum_{j=0}^{N=N} M^{*}(i, j) I(x+i, y+j)
\end{aligned}
$$

When the correlation function is evaluated at every pixel in the target image $\mathrm{I}(\mathrm{x}, \mathrm{y})$, the locations where the result is largest are those where the surrounding image is most similar to the model. The search algorithm then has to locate these peaks in the correlation result, and return their positions.

Unfortunately, ordinary correlation has the undesirable property that the result increases if the image simply gets brighter. The solution is to use a more complex, normalized version of the correlation function:

$$
r(x, y)=\frac{\sum_{i} \sum_{j}\left(M+\mathrm{x}+\mathrm{i}, \mathrm{y}+\mathrm{j}^{-}-\bar{M}\right)\left(I_{\mathrm{j}, \mathrm{j}}-\bar{I}\right)}{\sqrt{\sum_{i} \sum_{j}\left(M_{\mathrm{x}}+\mathrm{i}, \mathrm{y}+\mathrm{j}-\bar{M} \sum_{i} \sum_{j}\left(I_{\mathrm{i}, \mathrm{j}}-\bar{I}\right)\right.}}
$$

This expression has the desirable property that the result is unaffected by linear changes in the image or model pixel values.

Finally the result is converted to a percentage, where $100 \%$ represents a perfect match:

$$
\text { score }=\max (r, 0)^{2} * 100 \%
$$

\subsection{Acceptance Threshold}

To separate the results (match scores) of the normalized correlation an acceptance threshold is used. If the correspondence between the image and the model (also referred to as the match score) is less than this level, they are not considered as a match. The acceptance threshold depends on the noise and distortion of the image, however, poor quality images increase the chance of false matches and the search time (Fig. 6). The certainty threshold is the correlation value above which the algorithm can assume that is has found a match without continuing to search the rest of the image for a better one.

\subsection{Determination of Pan and Tilt Angles}

The pan and tilt angles of the camera with respect to the landmark are:

$$
\begin{gathered}
\varphi_{\text {pan }}=\operatorname{arctg} \frac{d_{p x}\left(C_{x}-u_{c}\right)}{f} \\
\varphi_{\text {tilt }}=\operatorname{arctg} \frac{d_{p y}\left(v_{c}-C_{y}\right)}{f}
\end{gathered}
$$

where $f$ is the focal distance, $\left(u_{c}, v_{c}\right)$ the center of the landmark, $\left(C_{x}, C_{y}\right)$ the center of the image and $d_{p x}, d_{p y}$ the cell size.

\section{Relocalization}

In the classic treatment for mobile robots localization, the position is predicted from the control orders, in our case, the results of the odometry are used to obtain the control orders that would produce the said results. The robot's pilot is based on reactive control, and by definition of this type of control, what the robot is going to do between two instants in time can not be known. Nevertheless, there was a need of ponderability between different measurements and the Kalman Filter provides with it [2][12].

Two equations are necessary for the Extended Kalman Filter. One that models the movement of the robot (transition function) and another for the measurement of the sensorial system (observation equation).

\subsection{System Model}

The equation of the state transition describes how the position of the vehicle changes from the previous state, $\mathrm{x}(\mathrm{k})=\left[\mathrm{x}_{\mathrm{k}}, \mathrm{y}_{\mathrm{k}}, \Theta_{\mathrm{k}}\right]^{\mathrm{T}}$, at time $\mathrm{t}_{\mathrm{k}}$ to $\mathrm{x}(\mathrm{k}+1)$ in $\mathrm{t}_{\mathrm{k}+1}$ due to a control action $\mathrm{u}(\mathrm{k})$ and the presence of a white noise $v(k)$ :

$$
\begin{array}{r}
x(k+1)=f(x(k), u(k))+v(k) \\
v(k) \approx N(0, Q(k))
\end{array}
$$

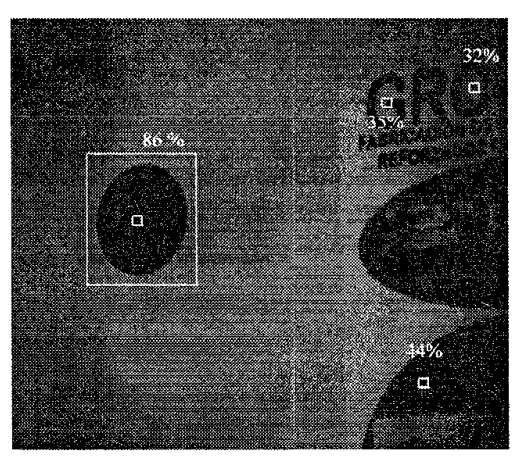

Figure 6. Landmark and match score. 
The control input $u(k)=u\left(T(k), \Delta \Theta_{1}(k), \Delta \Theta_{2}(k)\right)$, is a rotation $\Delta \Theta_{1}(k)$, followed by a translation of distance $T(k)$ and a new rotation $\Delta \Theta_{2}(k)$.

The function of state transition is described as:

$$
f(x(k), u(k))=\left[\begin{array}{c}
x(k)+T(k) \cos \Theta(k) \\
y(k)+T(k) \sin \Theta(k) \\
\Theta(k)+\Delta \Theta_{1}(k)+\Delta \Theta_{2}(k)
\end{array}\right]
$$

\subsection{Measurement Model}

The observation function depends on the position of the vehicle, the position of the landmark, and the kind of sensor. This function has the following expression:

$$
\begin{aligned}
& z_{j}(k)=h_{t}\left(p_{t}, x(k)\right)+w_{j}(k) \\
& w_{j}(k) \approx N(0, R(k))
\end{aligned}
$$

where $p=\left(p_{x}, p_{y}, p_{z}\right)^{T}$ is the position of the landmark. The observation function $h_{t}\left(p_{t}, x(k)\right)$ takes the form:

$$
\begin{gathered}
\phi_{\text {pan }}=\operatorname{arcsen}\left(\frac{y_{a t}-p_{y}}{\sqrt{\left(x_{a t}-p_{x}\right)^{2}+\left(y_{a t}-p_{y}\right)^{2}}}\right) \\
\phi_{\text {tilt }}=\operatorname{arctg}\left(\frac{p_{z}-z_{a t}}{\sqrt{\left(x_{a t}-p_{x}\right)^{2}+\left(y_{a t}-p_{y}\right)^{2}}}\right)
\end{gathered}
$$

Where $\left(\mathrm{x}_{\mathrm{at}}, \mathrm{y}_{\mathrm{at}}, \mathrm{z}_{\mathrm{at}}\right)$ is the position of the pan-tilt platform:

$$
\begin{aligned}
& x_{a t}=x_{k}+z_{a t} \cos \left(\Theta_{k}+C t e_{\Theta}\right) \\
& y_{a t}=y_{k}-z_{a t} \operatorname{sen}\left(\Theta_{k}+C t e_{\Theta}\right)
\end{aligned}
$$

\subsection{Localization Cycle}

Given the localization in an instant and known the information of the dead-reckoning in the subsequent instant, what should be perceived is compared with what

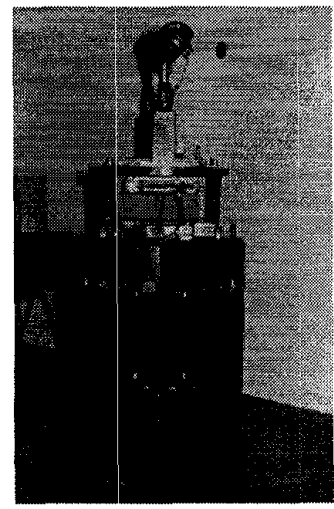

Figure 7. B21 Mobile robot. the sensor capture; by comparing both items of information, a new position and orientation of the robot is determined. The algorithm consists of the following steps: prediction of the position, prediction of measurements, observation, correspondence and estimation.

The final stage consists of using the correspondence confirmed between the predicted and the observed to calculate the new estimation of the robot position.

\section{Experimental Results}

All experiments have been conduced on a B21-RWI mobile vehicle (Fig. 7), equipped with a CCD camera (Sony, Ernitec 8-48 mm), mounted on a motorized TRC pan-tilt platform. The platform is able to perform tilt movements in a range of $0 \pm 90^{\circ}$, and pan movements in a range of $0 \pm 180^{\circ}$ (two different motors performs the movements), with an image acquisition board (MATROX Image-LC).

To test the localization method we made the mobile robot to move along different paths. To obtain the real position of the robot, a Geodimeter 600 (Theodolite) was used. The accuracy of distance measurement is $\pm 5 \mathrm{~mm}$. The maximum velocity of the robot was set at $30 \mathrm{~cm} / \mathrm{s}$. The experiments were performed in a $12 \times 7 \mathrm{~m}^{2}$ room, in which 6 landmarks had been placed.

This paper describes a class of algorithms for establishing a relocalization plan for a given path in a known environment. The relocalization plan consists of sets of landmarks that are visible from and must be detected at different points of the given path.

In the following figures, real situations are shown: in fig. 8, the mobile robot estimates its current position by dead-reckoning with wheel encoders. The position of the robot was calculated every $200 \mathrm{msec}$ during the navigation. The final error is about $22 \mathrm{~cm}$ in position and $3.5^{\circ}$ in orientation.

Fig. 9 shows the results of the position estimation process, in this case the artificial landmarks are placed around the usual path and are used to update the pose (position and orientation) of the vehicle, and to reset

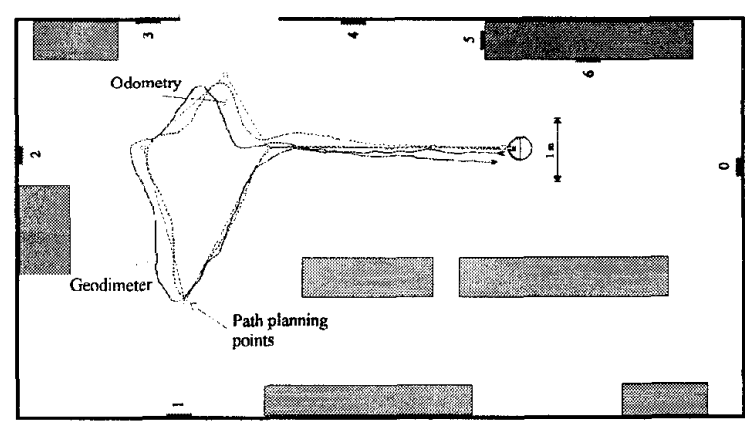

Figure 8. Position estimation by odometry. 


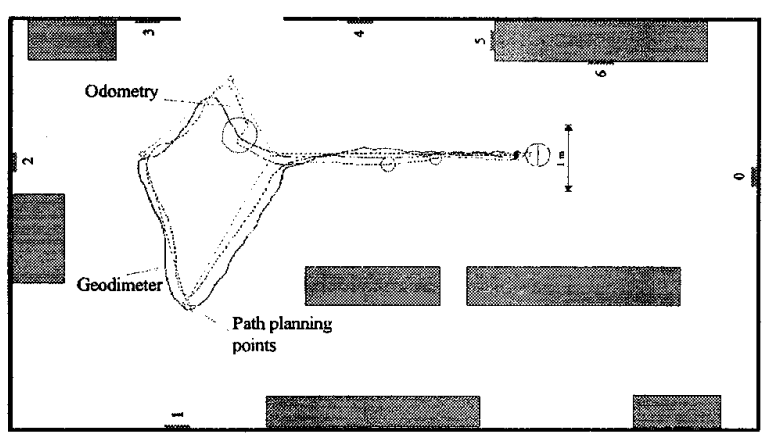

Figure 9. Relocalization process.

accumulated errors from odometry. The relocalization points are marked with a discontinuous circle. The final error is about $2 \mathrm{~cm}$ for position and $0.5^{\circ}$ for orientation. The filter convergence is nearly immediate, the updated position being the same after further iterations of the filtering. Note that a good localization was obtained by using one single landmark, but not always the same one.

Table 1 shows the list control areas, together with the list of landmarks selected and their suitability after the relocalization planning process.

\begin{tabular}{ccc}
\hline Control area & Visible landmarks & Suitability \\
\hline 0 & $0,4,6$ & $76 \%$ landmark 6 \\
1 & 4 & $63 \%$ landmark 4 \\
2 & $1,2,3$ & $93 \%$ landmark 2 \\
3 & $1,2,3$ & $93 \%$ landmark 3 \\
4 & $2,3,4$ & $97 \%$ landmark 2 \\
5 & 4 & $35 \%$ landmark 4 \\
6 & 0,6 & $41 \%$ landmark 0 \\
\hline
\end{tabular}

\section{Conclusions}

We have experimented the proposed localization method to find the position and heading angle of a mobile robot using the image of artificial landmarks. Through the experiments it is found that, when the camera looks at the landmark with a high vision angle, the errors of the $X, Y$ and $\Theta$ position tend to increase. The localization method developed in this paper proves to be computationally efficient and accurate enough for a mobile robot running in an indoor environment with no special lighting.

\section{Acknowledgments}

The authors gratefully acknowledge the funds provided by the Spanish Government through the CICYT projects TAP94-711 and TAP96-657, and thank B.J. Carabaño and M.J. López for their experimental work with the planner using the $\mathrm{B} 21$ robot.

\section{References}

[1] Bauer, R. (1995) "Active Manoeuvres for supporting the localization process of an autonomous mobile robot". Robotics and Autonomous Systems, vol 16, pp 39-46.

[2] Bar-Shalom, Y.; Fortmann, T. E. (1988) "Tracking and data association". Academic Press.

[3] Chenavier F., Crowley J., (1992) "Position estimation for a mobile robot using vision and odometry" . IEEE Int Conf. on Rob. and Aut. pp 2631-37, Nice-France.

[4] Deng, X., Milios, E.; (1996) "Landmark selection strategies for path execution". Robotics and Autonomous Systems, vol 17, pp 171-185.

[5] D'Orazio, T., Lovergine, F.P., Ianigro, M., Stella, E. and Distante, A. (1994) "Mobile Robot Position Determination Using Visual Landmarks". IEEE Transactions on Industrial Electronics. December, pp 654-662.

[6] Escalera,A.; Moreno, L.; Salichs,M.A.; Armingol, J.M.; (1996) "Continuous mobile robot localization by using structured light and a geometric map". International Journal of Systems Science, Vol 27, $n^{\circ} 8$, pp 771-782.

[7] Garibotto G.B., Masciangelo S. (1992) "3D computer vision for navigation/control of mobile robots". Machine Perception(AGARD-LS-185) pp 5/1-13, Madrid.

[8] Han M.; Rhee, S.; (1994) "Navigation control for a mobile robot". Journal of Robotics Systems 11 (3), pp 169-179.

[9] Kanbara T., Miura, J.; (1993) "Selection of efficient landmarks for an autonomous vehicle". Proceedings of the 1993 IEEE/RSJ. International Conference on Intelligent Robots and Systems, pp 26-30.

[10] Lazanas, A., Latombe, J.C.; (1992) "Landmark-Based Robot Navigation". 10th National Conf. on Artificial Intelligence (AAAI'92), San José (USA).

[11] Lazanas, A., Latombe, J.C.; (1995) "Motion planning with uncertainty: a landmark approach". Artificial Intelligence 76, pp 287-317.

[12] Leonard, J.J.; Durrant-White H.F.; (1992) "Directed sonar sensing for mobile robot navigation". Kluwer Academic Publishers.

[13] Moreno, L., Salichs, M.A., Gachet, D., Pimentel, J.; (1995) "Neural networks for mobile robot piloting control". Applications of Artificial Neural Networks in Robotic Control. Prentice Hall. pp 137-161.

[14] Ricotti, M., Liotta, A. (1995) "Real Time landmarks detection for the Mobile Robot PARIDE". Proceedings of the SPIE, vol 2423, pp 60-71.

[15] Salichs, M.A., Gachet, D., Moreno, L., Pimentel, J.; (1994) "Learning and Control in Autonomous Systems". AIRTC'94. Symposium on Artificial Intelligent in Real Time Control. 